\title{
Immunostaining Protocol: P-Stat3 (Xenograft and Mice)
}

Alexandre Calon ${ }^{1}$, Elisa Espinet ${ }^{1}$, Sergio Palomo-Ponce ${ }^{1}$, Daniele

V. F. Tauriello ${ }^{1}$, Mar Iglesias ${ }^{2}$, María Virtudes Céspedes ${ }^{3}$, Marta Sevillano ${ }^{3}$, Cristina Nadal ${ }^{4}$, Peter Jung ${ }^{4}$, Xiang H.-F. Zhang ${ }^{5}$, Daniel Byrom ${ }^{6}$, Antoni Riera ${ }^{6}$, David Rossell ${ }^{7}$, Ramón Mangues $^{7}$, Joan Massague ${ }^{7}$, Elena Sancho ${ }^{7^{*}}$ and Eduard Batlle ${ }^{7^{*}}$

${ }^{1}$ Oncology Programme, Institute for Research in Biomedicine, Barcelona, Spain; ${ }^{2}$ Department of Pathology, Hospital Universitari del Mar, Barcelona, Spain; ${ }^{3}$ IIB Sant Pau, Hospital de la Santa Creu i Sant Pau, Barcelona, Spain; ${ }^{4}$ Institut de Malalties Hemato-Oncològiques, Hospital Clínic, Barcelona, Spain; ${ }^{5}$ Cancer Biology and Genetics Program, Memorial Sloan-Kettering Cancer Center, New York, USA; ${ }^{6}$ Chemistry and Molecular Pharmacology Programme, Institute for Research in Biomedicine, Barcelona, Spain; ${ }^{7}$ Biostatistics and Bioinformatics Unit, Institute for Research in Biomedicine, Barcelona, Spain

*For correspondence: elena.sancho@irbbarcelona.org; eduard.batlle@irbbarcelona.org

[Abstract] We sought to understand the mechanisms behind the potent effect of stromal TGFbeta program on the capacity of colorectal cancer (CRC) cells to initiate metastasis. We discovered that mice subcutaneous tumors and metastases generated in the context of a TGFbeta activated microenvironment displayed prominent accumulation of p-STAT3 in CRC cells compared with those derived from control cells. STAT3 signaling depended on GP130 as shown by strong reduction of epithelial p STAT3 levels upon GP130 shRNA-mediated knockdown in CRC cells.

\section{Materials and Reagents}

1. Paraffin sections (subcutaneous tumors samples or liver metastasis from nude mice respectively injected subcutaneously or intrasplenic with CRC cells)

2. XILOL

Note: Xylol also referred to as xylene or dimethylbenzene is a solvent used in histology as a clearing agent to remove paraffin from dried microscope slides prior to staining.

3. MilliQ $\mathrm{H}_{2} \mathrm{O}$

4. Wash buffer (Dako, catalog number: K800721)

5. Rabbit anti-P-Stat3 (Cell Signaling Technology, catalog number: 9145S)

6. BrightVision poly-HRP anti- Rabbit (Immunologic, catalog number: DPVR110HRP)

7. Envision FLEX antibody diluent (Dako, catalog number: K8006)

8. Peroxidase Blocking Solution (Dako, catalog number: S202386) 
9. ImmPACT DAB (Vector Laboratories, catalog number: SK-4105)

10. DPX mounting media (Sigma-Aldrich, catalog number: 06522)

11. Hematoxylin

12. Tris/EDTA (pH 9.0) (see Recipes)

\section{Equipment}

1. Oven

2. Immunostaining apparatus

\section{Procedure}

1. Stove samples at $65{ }^{\circ} \mathrm{C}$ just before starting the immunostaining technique. Remove the samples from the oven when the wax present in sections is completely undone.

2. De-waxing and rehydration: Place slides in a rack to perform following washes (bath).
a. XILOL: $10 \mathrm{~min}$
b. XILOL: $10 \mathrm{~min}$
c. XILOL: 5 min
d. $100 \% \mathrm{EtOH}: 10 \mathrm{~min}$
e. $100 \% \mathrm{EtOH}: 5$ min
f. $96 \% \mathrm{EtOH}: 5 \mathrm{~min}$
g. $90 \% \mathrm{EtOH}: 10-15$ times
h. $80 \% \mathrm{EtOH}: 10-15$ times
i. $70 \% \mathrm{EtOH}: 10-15$ times
j. $\quad 50 \%$ EtOH: 10-15 times
k. $25 \% \mathrm{EtOH}: 10-15$ times
I. $\mathrm{H}_{2} \mathrm{O}$ MilliQ: 10-15 times

3. Antigen retrieval.
a. Tris-EDTA Buffer ( $\mathrm{pH} 9.0)$
b. Time: $20 \mathrm{~min}$ in boiling water

4. 3 washes 5 min with $1 \mathrm{ml} 1 \times$ wash buffer.

5. Blocking endogenous peroxidase.
a. $200 \mathrm{ml}$ Peroxidase Blocking Solution
b. Time: $10 \mathrm{~min}$

6. 3 washes $5 \mathrm{~min}$ with $1 \mathrm{ml} 1 \mathrm{x}$ wash buffer.

7. Incubation with primary antibody.
a. Antibody: Rabbit anti-P-Stat3 
b. Dilution 1/200 in Envision FLEX antibody diluent

c. $200 \mu \mathrm{l} / \mathrm{sample}$

d. $\mathrm{O} / \mathrm{N} 4{ }^{\circ} \mathrm{C}$

8. 3 washes 5 min with $1 \mathrm{ml} 1 \times$ wash buffer

9. Incubation with antibody BrightVision
a. Antiboby: BrightVision anti-Rabbit
b. $150 \mu \mathrm{l} / \mathrm{sample}$
c. Time: $45 \mathrm{~min}$ at room temperature

10. 3 washes 5 min with $1 \mathrm{ml} 1 \times$ wash buffer

11. Revealed with ImmPACT DAB.
a. $200 \mu \mathrm{l} / \mathrm{sample}$
b. Time: $10 \mathrm{~min}$

12. 3 washes $5 \mathrm{~min}$ with $1 \mathrm{ml}$ distilled water.

13. Hematoxylin (1 ml) counterstaining, time: 2 min.

14. Rinse in distilled water bath.

15. Rinse in TAP water bath.

16. Dehydration and mounting with DPX.

\section{$\underline{\text { Representative data }}$}

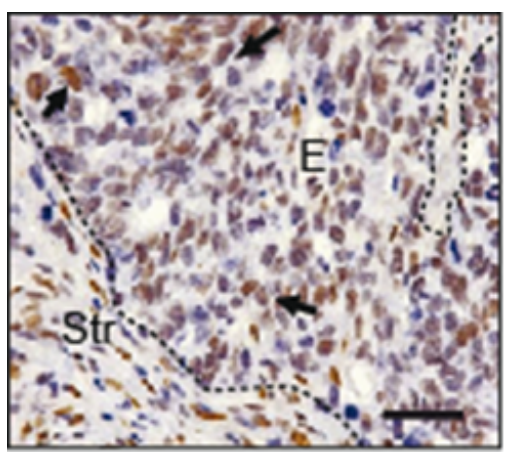

Figure 1. p-STAT3 staining of liver metastases generated after intrasplenic injection of CRC cells. Note strong staining in epithelial cells (arrows). E: epithelial cells, Str: stromal cells. Scale bar $=50 \mu \mathrm{m}$.

\section{$\underline{\text { Recipes }}$}

1. Tris/EDTA (pH 9.0)

$12 \mathrm{~g}$ Tris

$3.7 \mathrm{~g}$ EDTA in $10 \mathrm{~L}$ MilliQ 


\section{Acknowledgments}

A.C. and D.V.F.T hold a Juan de la Cierva postdoctoral fellowship, E.E. an FPI PhD fellowship (both from Spanish Ministry of Science and Innovation). This work has been supported by grants from Instituto de Salud Carlos III FEDER (RD09/0076/00036) and the "Xarxa de Bancs de tumors sponsored by Pla Director d'Oncologia de Catalunya (XBTC), to E.B. from the European Research Council (Starting grant - 208488) and Consolider programmes (MICINN), to E.S. and A.C. by the Spanish Ministry of Science and Innovation, to JM by NIH grant CA34610 and to RM by grants PS09/00965 (MICINN) and NanoCoMets (CIBERBBN).

\section{References}

1. Calon, A., Espinet, E., Palomo-Ponce, S., Tauriello, D. V., Iglesias, M., Cespedes, M. V., Sevillano, M., Nadal, C., Jung, P., Zhang, X. H., Byrom, D., Riera, A., Rossell, D., Mangues, R., Massague, J., Sancho, E. and Batlle, E. (2012). Dependency of colorectal cancer on a TGF-beta-driven program in stromal cells for metastasis initiation. Cancer Cell 22(5): 571-584. 